\title{
Suppression of Foxo3-Gatm by miR-132-3p Accelerates Cyst Formation by Up-Regulating ROS in Autosomal Dominant Polycystic Kidney Disease
}

\author{
Seonju Choi ${ }^{\dagger}$, Do Yeon $\mathrm{Kim}^{\dagger}$, Yejin Ahn ${ }^{\dagger}$, Eun Ji Lee and Jong Hoon Park* \\ Department of Biological Sciences and Research Institute of Women's Health, Sookmyung Women's University, Seoul 04310, \\ Republic of Korea
}

\begin{abstract}
Accumulation of reactive oxygen species (ROS) is associated with the development of various diseases. However, the molecular mechanisms underlying oxidative stress that lead to such diseases like autosomal dominant polycystic kidney disease (ADPKD) remain unclear. Here, we observed that oxidative stress markers were increased in Pkd1/ff:HoxB7-Cre mice. Forkhead transcription factors of the $\mathrm{O}$ class (FOXOs) are known key regulators of the oxidative stress response, which have been observed with the expression of FoxO3a in an ADPKD mouse model in the present study. An integrated analysis of two datasets for differentially expressed miRNA, such as miRNA sequencing analysis of $P k d 1$ conditional knockout mice and microarray analysis of samples from ADPKD patients, showed that miR-132-3p was a key regulator of FOXO3a in ADPKD. miR-132-3p was significantly upregulated in ADPKD which directly targeted FOXO3 in both mouse and human cell lines. Interestingly, the mitochondrial gene Gatm was downregulated in ADPKD which led to a decreased inhibition of Foxo3. Overexpression of miR-132-3p coupled with knockdown of Foxo3 and Gatm increased ROS and accelerated cyst formation in 3D culture. This study reveals a novel mechanism involving miR-132-3p, Foxo3, and Gatm that is associated with the oxidative stress that occurs during cystogenesis in ADPKD.
\end{abstract}

Key Words: ADPKD, FOXO3, ROS, MicroRNA, Cystogenesis

\section{INTRODUCTION}

Autosomal dominant polycystic kidney disease (ADPKD) is the most common hereditary renal disorder and a leading cause of genetic end-stage renal disease (Igarashi and Somlo, 2007). It affects one in thousand individuals and is caused by mutations in either PKD1 ( $85 \%$ of the patients) or PKD2 ( 15\% of the patients), which encode polycystin 1 and 2, respectively (Peters and Sandkuijl, 1992; Mochizuki et al., 1996). Tolvaptan, a vasopressin V2 receptor blocker, has been licensed for the treatment of ADPKD in several countries. However, because of its limited efficacy and side effects, the need for alternative approaches remains (Torres et al., 2012; Gansevoort et al., 2016). Recent clinical studies have reported that oxidative stress occurs in the early stage of ADPKD and worsens with disease progression; however, molecular mechanisms underlying this phenomenon remain unclear (Andries et al., 2018).

Forkhead box protein $\mathrm{O}$ (FOXO) transcription factors, including FOXO1 (FKHR), FOXO3 (FOXO3a, FKHRL1), FOXO4 (AFX), and FOXO6 in mammals (Calnan and Brunet, 2008), are negatively regulated by the phosphatidylinositol 3-kinase (PI3K)/serine-threonine kinase (AKT) signaling pathway. This AKT-mediated phosphorylation of FOXO results in its exportation into the cytoplasm and enhancement of proteasomal degradation (Tzivion et al., 2011). These FOXO transcription factors regulate the expression of genes involved in various cellular processes such as the cell cycle, apoptosis, and reactive oxygen species (ROS) detoxification (Kops et al., 2002; van der Horst and Burgering, 2007; Ham et al., 2019). Within the cell, mitochondria are known to be major sources of ROS; superoxide $\left(\mathrm{O}_{2}{ }^{-}\right)$, an early product of the mitochondrial respi-

\section{Open Access https://doi.org/10.4062/biomolther.2020.197}

This is an Open Access article distributed under the terms of the Creative Commons Attribution Non-Commercial License (http://creativecommons.org/licenses/by-nc/4.0/) which permits unrestricted non-commercial use, distribution, and reproduction in any medium, provided the original work is properly cited.
Received Nov 3, 2020 Revised Nov 24, 2020 Accepted Nov 25, 2020 Published Online Jan 7, 2021

\section{*Corresponding Author}

E-mail: parkjh@sookmyung.ac.kr

Tel: +82-2-710-9414, Fax: +82-2-2077-7258

${ }^{\dagger}$ The first three authors contributed equally to this work. 
ratory chain, can be rapidly converted to $\mathrm{H}_{2} \mathrm{O}_{2}$ by superoxide dismutase (SOD) and then reduced to $\mathrm{H}_{2} \mathrm{O}$ by catalase or glutathione peroxidase (Zhang et al., 2016). Notably, FOXO3 transcriptionally regulates the antioxidant enzymes SOD2 and catalase (Liu et al., 2005). Although the FOXO3 has been reported that induce a number of ROS related genes (Hagenbuchner et al., 2012), the role of FOXO3 and the relationship between FOXO3 and ROS in ADPKD remain unresolved.

In this study, we aimed to identify novel mechanisms associated with oxidative stress and cystogenesis in ADPKD. We observed that Foxo3a was a key regulator of ROS accumulation in a Pkd1 conditional knockout (cKO) mouse model. Through integrated analysis of miRNA-seq and microarray data from PKD model mice and ADPKD patients, respectively, Foxo3a was revealed to be suppressed by miR-132-3p in an ADPKD model. We also identified a novel downstream target, Gatm, that affected ROS production and cystogenesis. Therefore, we propose that miR-132-3p-mediated signaling is involved in ADPKD and that investigation on their associated mechanisms is essential to identify novel therapeutic targets.

\section{MATERIALS AND METHODS}

\section{Mice}

$P k d 1^{\text {flox}: H o x B 7-C r e ~ m i c e ~ w e r e ~ u s e d ~ i n ~ t h i s ~ s t u d y . ~ T o ~ g e n e r a t e ~}$ a $P k d 1$ conditional-KO mouse model, we used $P k d 1^{\text {flox }}$ mice in combination with HoxB7-Cre transgenic mice, and the phenotype of the obtained mice was previously characterized (Woo et al., 2017). The Pkd1 $1^{\text {flox }}$ mice were obtained from Dr. Stefan Somlo at Yale University, and the HoxB7-Cre transgenic mice, which specifically express Cre recombinase in the kidney collecting ducts, were obtained from Minho Shong at Chungnam National University. For genotyping, the Pkd1/f/f alleles and HoxB7-cre transgene were PCR amplified from tail DNA using the following primers: Pkd1/f/f forward 5'-CCGCTGTGTCTCAGTGCCTG-3' and reverse 5'-CAAGAGGGCTTTTCTTGCTG-3'; HoxB7-cre transgene forward 5'-GCGGTCTGGCAGTAAAAACTATC-' and reverse 5'-GTGAAACAGCATTGCTGTCACTT-3'. All experimental procedures were performed in accordance with relevant guidelines and regulations on laboratory animal care. All experimental protocols were reviewed and approved by the IACUC of Sookmyung Women's University (Seoul, Korea).

\section{Human specimens}

This study was conducted in accordance with the Declaration of Helsinki and was approved by the Institutional Review Board of Seoul National University Hospital (Seoul, Korea) (H-0701-033-195). Informed consent was obtained from all patients. Renal cystic tissues were obtained from the renal cortex surrounding the cysts of patients with ADPKD while undergoing nephrectomy. Non-ADPKD renal tissue were obtained from patients with clear cell renal cell carcinoma (RCC) as a control, and malignant cell infiltration was excluded by histology. All these tissues were also used in our previous study (Woo et al., 2014; Kim et al., 2019).

\section{Cell culture}

Inner medullary collecting duct cells (IMCD cells; CRL$2123^{\mathrm{TM}}$, ATCC, Manassas, VI, USA) were cultured in DMEM/ F12 medium (LM002-04, Welgene, Gyeongsan, Korea) containing $10 \%$ foetal bovine serum (FBS; 26140-079, Gibco,
Waltham, MA, USA), and $1 \%$ penicillin-streptomycin (LS 202-02, Welgene). Normal human renal cortical epithelial cells (HRCE; CC-2554, LONZA, Morristown, NJ, USA) were cultured in REGM medium (CC-4190, LONZA) containing 1\% penicillin-streptomycin. WT9-7 (CRL-2830 ${ }^{\mathrm{TM}}$, ATCC), a proximal cortical tubule epithelial cell line isolated from renal cysts from a patient with ADPKD, was cultured in DMEM (LM001-05, Welgene) containing 10\% FBS and 1\% penicillin-streptomycin in culture dishes coated with type I bovine collagen (\#354231, Corning, New York, NY, USA). Human embryonic kidney 293T cells (HEK293T) were cultured in DMEM (LM001-05, Welgene) containing $10 \%$ FBS and $1 \%$ penicillin-streptomycin in Poly-D-lysine-coated dishes (P7280, Sigma-Aldrich, St. Louis, $\mathrm{MO}$, USA). These cells were grown in a humidified $5 \% \mathrm{CO}_{2}$ incubator at $37^{\circ} \mathrm{C}$. For hypoxic conditions, cells were incubated in $5 \% \mathrm{CO}_{2}$ and $1 \% \mathrm{O}_{2}$ balanced with $\mathrm{N}_{2}$ in a hypoxic chamber within an incubator at $37^{\circ} \mathrm{C}$.

\section{Total RNA isolation and miRNA expression analysis}

Total RNA was extracted from cell lines and kidney tissues using the miRNeasy Mini Kit (Qiagen, Hilden, Germany) according to the manufacturer's instructions. To assess miRNA expression, first, cDNA was prepared from total RNA $(1 \mu \mathrm{g})$ using the miScript II RT kit (Qiagen) according to the manufacturer's protocol. Then, quantitative real-time PCR was performed using miScript Primer Assays (Qiagen) and the miScript SYBR Green PCR Kit (Qiagen) according to the manufacturer's protocol. RNU6 was used as an endogenous miRNA control.

\section{Luciferase reporter assay}

The mouse and human FOXO3 3'-UTR were amplified by PCR using RBC Taq DNA Polymerase (RBC Bioscience, New Taipei, Taiwan). The sequence of miR-132-3p was mutated using a PCR-based approach. The sequences of the primers used to amplify the wild-type and mutant FOXO3 3'-UTR were as follows: mouse wild-type forward 5'-CTAGTTGTTTAAACGAGCTCGCAGAGACTGTTAGCAGC-3', mouse mutant forward 5'-CTAGTTGTTTAAACGAGCTCGCAGAGCAGTGTAGCAGC-3', mouse wild-type and mutant reverse 5'-CGACTCTA GACTCGAGCACAAGACGACACTTAAAATA-3', human wildtype forward 5'-CTAGTTGTTTAAACGAGCTCGCAGAGACTGTTAATGGCC-3', human mutant forward 5'-CTAGTTGTTTAAACGAGCTCGCAGAGCAGTGTAATGGC-3', and human wild-type and mutant reverse 5'-CGACTCTAGACTCGAGCTTAAAACGTCCCATAAACC-3'. The amplified PCR fragments were cloned into the $\mathrm{Sacl}$ and $\mathrm{Xhol}$ restriction sites of the pmiRGLO vector (Promega, Madison, WI, USA) using the InFusion HD Cloning kit (Takara Bio, Inc., Kusatsu, Japan). For transfection, IMCD cells $\left(2 \times 10^{4}\right)$ and HEK293T cells $\left(3 \times 10^{4}\right)$ were plated into 12-well plates and co-transfected with wildtype or mutant 3'-UTR constructs $(750 \mu \mathrm{g})$ and negative control mimics or miR-132-3p mimics (20 nM; Ambion, Waltham, MA, USA) one day after seeding using Lipofectamine 2000 (Invitrogen, Carlsbad, CA, USA) according to the manufacturer's instructions. Luciferase activity was measured $48 \mathrm{~h}$ after transfection using the Dual Luciferase Assay system (Promega). All transfection experiments were performed in triplicate. Serial deletion fragments of the Gatm promoter region (p0.2kb, p0.4kb, p0.7kb, and p0.8kb) were amplified by PCR and cloned into the Sacl and Nhel restriction sites of the pGL3-basic vector (Promega). The following primers were 
used to generate the serial deletion constructs: p0.2kb forward 5'-TATCGATAGGTACCGAGCTCGGAGGCACGAGCTAAAAAAAAAAAA-3', p0.4kb forward 5'-TATCGATAGGTACCGAGCTCCATAGGCTCAGGTCGACAAACTTG-3', p0.7kb forward 5'- TATCGATAGGTACCGAGCTCCTGGACGACACAGCCAGGC-3', p0.8kb forward 5'- TATCGATAGGTACCGAGCTCGCATTTTAAGAACAGAGC-3', and reverse 5'- TCGAGCCCGGGCTAGCCGCCCCGTCTCTGCTGCT-3'. Luciferase activity was measured in the same manner as described for the $3^{\prime}$-UTR luciferase assay.

\section{Transfection}

miRNA mimics (Ambion) or inhibitors (Dharmacon, Lafayette, LA, USA) of miR-132-3p were reverse transfected into cell lines (at $30 \mathrm{nM}$ ) using siPORT NeoFX transfection reagent (Ambion) for $48 \mathrm{~h}$. For the control experiments cells were transfected with a negative control miRNA mimic (mirVana ${ }^{\mathrm{TM}}$ miRNA Inhibitor, Negative Control \#1, Ambion) or the inhibitor (miRIDIAN microRNA Hairpin Inhibitor Negative Control \#1, Dharmacon). Small interfering RNAs (siRNAs) targeting the mouse or human Fox3 gene (Santa Cruz, Dallas, TX, USA) were transfected into cells (at $20 \mathrm{nM}$ ) using Lipofectamine RNAiMAX reagent (Invitrogen) according to the manufacturer's instructions. Subsequent experiments were performed 48 $\mathrm{h}$ after transfection.

\section{Quantitative real-time PCR (qRT-PCR)}

mRNA was isolated with the NucleoSpin ${ }^{\circledR}$ RNA/Protein kit (Macherey-Nagel, Duren, Germany) according to the manufacturer's instructions. For mRNA expression analysis, The isolated RNA $(1 \mu \mathrm{g})$ was reverse-transcribed to cDNA using M-MLV Reverse Transcriptase (Promega), RNase inhibitor (Promega), oligo-dT (100 nM), and dNTPs (2.5 mM; Promega). Quantitative real-time PCR was performed using qPCRBIO SyGreen Blue Mix (PCR Biosystem, London, UK) and a LightCycler ${ }^{\circledR} 96$ System (Roche, Basel, Switzerland) according to the manufacturer's instructions. Mouse $\beta$-actin and human 18S rRNA were used as endogenous controls.

\section{Western blot analysis}

Proteins were isolated using the NucleoSpin ${ }^{\circledR}$ RNA/Protein kit (Macherey-Nagel), according to the manufacturer's proto$\mathrm{col}$. The protein concentration was calculated using the BCA assay and bicinchoninic acid and copper (II) sulphate solutions (Sigma-Aldrich), and equal amounts of protein were separated by $8-12 \%$ SDS-PAGE and were electro-transferred to polyvinylidene fluoride (PVDF) membranes (ATTO, Tokyo, Japan). Immunoblots were probed with primary antibodies against the following proteins: FoxO3a (\#2497, Cell Signaling, Danvers, MA, USA), HIF-1 $\alpha$ (\#14179, Cell Signaling), GATM (ab228937, Abcam, Cambridge, MA, USA), ALDH4A1 (ab185208, Abcam), ALDH1L1 (sc-100497, Santa Cruz), ALDH6A1 (sc-271582, Santa Cruz), COX IV (ab33985, Abcam), $\alpha$-tubulin (\#3873, Cell Signaling), and $\beta$-actin (A300-491A, Bethyl Laboratories, Montgomery, AL, USA). The primary antibodies were diluted 1:1,000 with $1 \%$ skim milk in PBS containing $1 \%$ Tween $^{\circledR} 20$ (Sigma-Aldrich) and incubated at $4^{\circ} \mathrm{C}$ overnight. Horseradish peroxidase-conjugated secondary antibodies in $2 \%$ skim milk were incubated with the blots for $1 \mathrm{~h}$ at room temperature. Immunoreactive proteins were detected using chemiluminescence reagent EzWestLumi Plus (ATTO) and visualized with a LAS-3000 instrument (Fujifilm, Tokyo,
Japan).

\section{ROS detection by immunohistochemistry}

Kidneys were fixed in $4 \%$ paraformaldehyde overnight and then embedded in paraffin. Kidney tissue sections were deparaffinized by three changes of Histoclear II (National Diagnostics, Atlanta, GA, USA) and then rehydrated in a graded series of ethanol. For antigen retrieval, the sections were heated in a TintoRetriever Pressure Cooker (Bio SB, Santa Barbara, CA, USA) with Borg Decloaker RTU (Biocare Medical, Pacheco, CA, USA) and were incubated with UV Hydrogen Peroxide Block (Thermo Fisher Scientific, Waltham, MA, USA). Sections were blocked with blocking solution for $1 \mathrm{~h}$ at room temperature, and then incubated overnight with the appropriate primary antibodies at $4^{\circ} \mathrm{C}$. The primary antibodies used for immunostaining were anti-8-OHdG antibody (sc-66036, Santa Cruz) and anti-4 hydroxynonenal (ab48506, Abcam). Peroxidase activity was detected using the VECTASTAIN Elite ABC HRP Kit (PK-6200, Vector Labs, Burlingame, CA, USA) and VECTOR NovaRED Peroxidase (HRP) Substrate Kit (SK-4800, Vector Labs) according to the manufacturer's instructions. Slides were mounted with VectaMount Permanent Mounting Medium (H-5000, Vector Labs) and analysed.

\section{Mitochondrial ROS measurement}

Mitochondrial ROS levels were assessed using MitoSOX ${ }^{\mathrm{TM}}$ Red mitochondrial superoxide indicator (Invitrogen). MitoSOX $^{\mathrm{TM}}$ Red is a nonfluorescent dihydroethidium-based dye that is oxidized by mitochondrial superoxide and emits a red fluorescence. IMCD cells were grown on glass coverslips in six-well plates. After 2 days of incubation, the cells were incubated with MitoSOX ${ }^{\mathrm{TM}} \operatorname{Red}(5 \mu \mathrm{M})$ in HBSS/Ca/Mg (Gibco) for $10 \mathrm{~min}$ at $37^{\circ} \mathrm{C}$ and then washed three times with PBS. The cells were fixed with $4 \%$ paraformaldehyde at room temperature for 10 min, permeabilized with $0.2 \%$ Triton X-100 containing $1 \%$ bovine serum albumin for $10 \mathrm{~min}$. Nuclei were stained with DAPI. The slides were mounted with fluorescence mounting medium (Dako, Santa Clara, CA, USA), and images were obtained under a confocal laser scanning microscope (LSM700, Carl Zeiss, Oberkochen, Germany). The fluorescence intensity of MitoSOX TM Red was quantified using ImageJ software (NIH, Bethesda, MD, USA).

$1 \mathrm{mM}$ stock solutions of MitoTracker ${ }^{\mathrm{TM}}$ Green (Thermo Fisher Scientific) were diluted with DMEM/F12 to a $200 \mathrm{nM}$ working concentration and incubated with cells for $20 \mathrm{~min}$ at $37^{\circ} \mathrm{C}$. After that, $1 \mathrm{ul}$ of $5 \mathrm{mM}$ MitoSOX ${ }^{\mathrm{TM}}$ Red were added and incubated for $10 \mathrm{~min}$ at $37^{\circ} \mathrm{C}$.

\section{Three-dimensional (3D) cell culture}

IMCD cells $\left(4 \times 10^{4}\right.$ cells/well) were mixed with Matrigel (Corning) at a 1:1 ratio and plated in 8-well chamber slides. Culture medium was added after $1 \mathrm{~h}$ and replaced every day. Cells in Matrigel were grown in a humidified atmosphere containing $5 \% \mathrm{CO}_{2}$ at $37^{\circ} \mathrm{C}$ for $4-6$ days. Phase contrast images of individual cystic spheroids were obtained under a light microscope (IX70, Olympus, Tokyo, Japan) and ISP capture software (Olympus) after 4,5 , and 6 days in culture. The cyst area was measured using ImageJ software $(\mathrm{NIH})$.

\section{Mitochondrial fractionation}

Mitochondria and the cytosol were separated by using the Mitochondria Isolation Kit (Pierce Biotechnology, Waltham, 
A

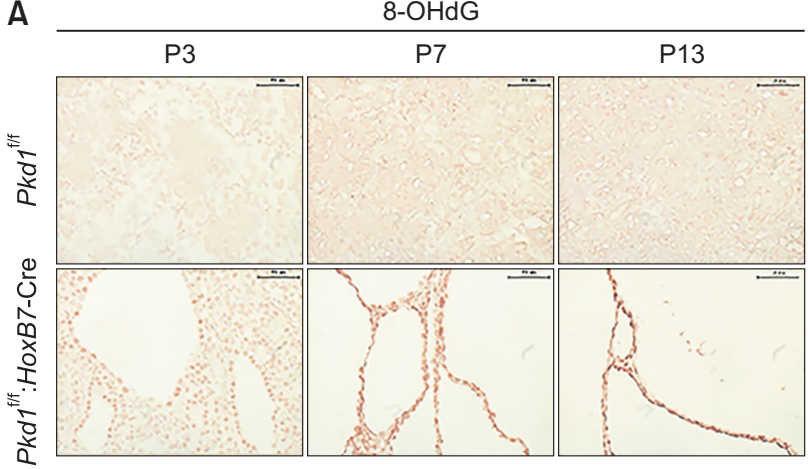

$\mathbf{E}$

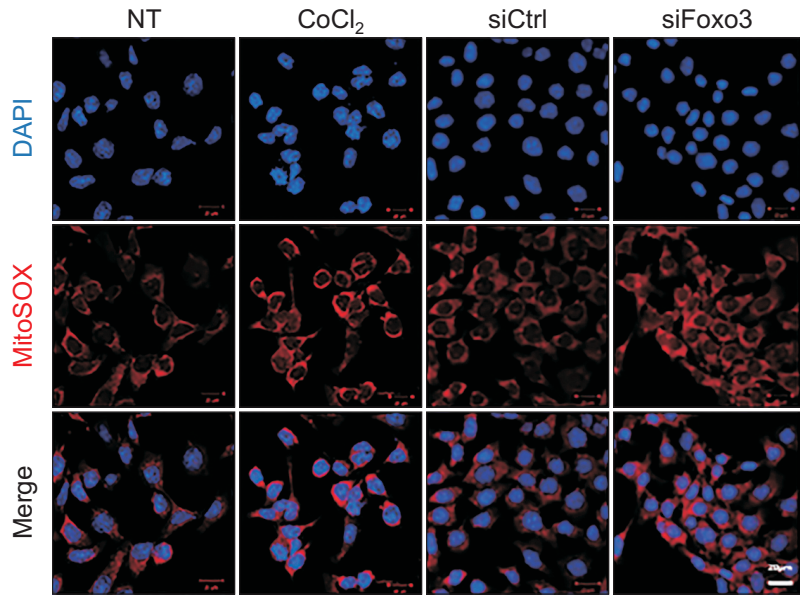

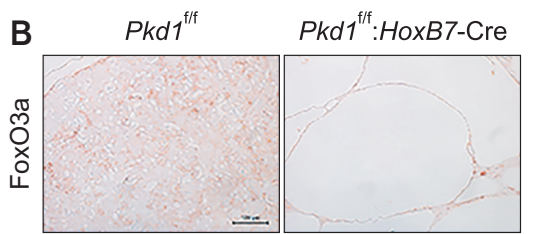

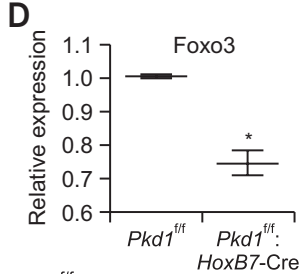

C

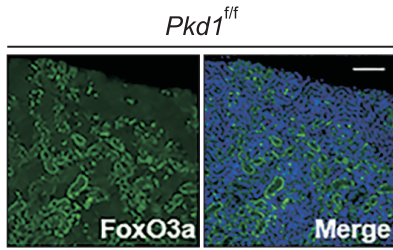

$P k d 1^{f / f}:$ HoxB7-Cre

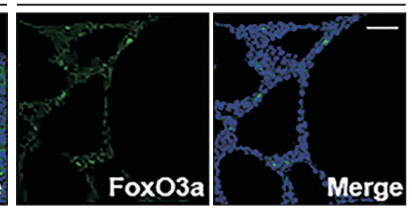

$\mathbf{F}$

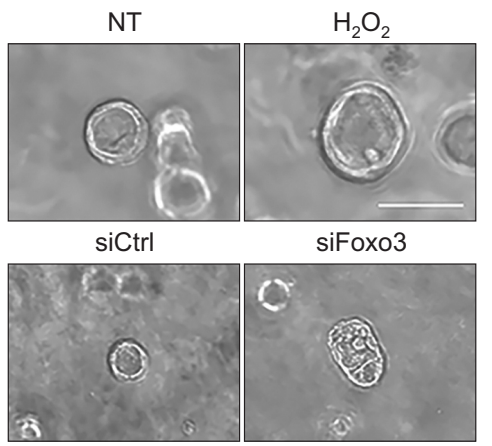

EV

Foxo3

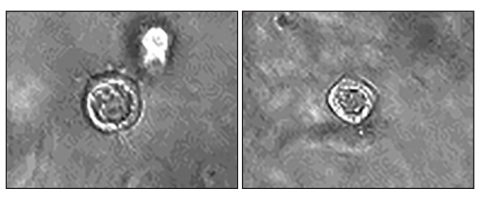

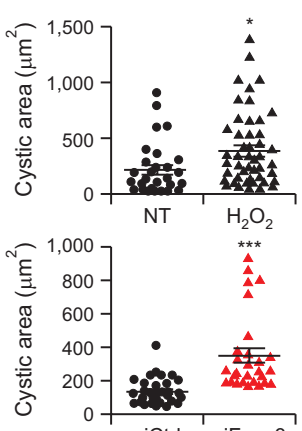

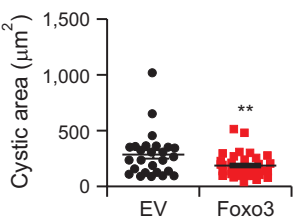

siCtrl siFoxo3

Fig. 1. Inhibition of Foxo3 increase mitochondrial superoxide levels and accelerate cyst enlargement. (A) Immunohistochemical staining of 8-OHdG, a DNA damage marker, in the kidney tissues of $P k d 1^{f / f}$ and $P k d 1^{f / f}$ :HoxB7-Cre mice on postnatal days 3, 7, and 13. Scale bar, $50 \mu \mathrm{m}$. (B, C) FoxO3a were detected by immunohistochemistry in $P k d 1^{\mathrm{fff}}$ and $P k d 1^{\mathrm{fff}}$ :HoxB7-Cre mice on P7. Scale bar, $100 \mu \mathrm{m}$ on panel B and $50 \mu \mathrm{m}$ on panel C. (D) The transcript level of Foxo3 in $P k d 1^{t / f}$ and $P k d 1^{t / f}$ :HoxB7-Cre mice on P7. (E) MitoSOX staining to detect mitochondrial superoxide $\left(\mathrm{O}_{2}^{-}\right)$levels by confocal microscopy $(400 \times$ magnification). $\mathrm{CoCl} 2$ was used as a positive control. NT, non-treated. Nuclei were stained with DAPI (blue). $(\mathrm{F})$ Cyst formation in $\mathrm{H}_{2} \mathrm{O}_{2}$ - or non-treated mIMCD cells in a Matrigel 3D culture system. Cyst area was measured after 4 days in 3D culture. Cyst formation in mIMCD cells transfected with Foxo3 siRNA or Foxo3 vector and control in a Matrigel 3D culture system. Cyst size was measured after 5 days in 3D culture. Scale bar, $50 \mu \mathrm{m}$. Data are the mean \pm SD of triplicate samples from three independent experiments. ${ }^{*} p<0.05,{ }^{* *} p<0.01,{ }^{* * *} p<0.001$.

MA, USA) according to the manufacturer's instructions. The mitochondrial fraction was confirmed using an anti-COX IV antibody (ab33985, Abcam). The cytosolic fraction was confirmed using an $\alpha$-tubulin antibody (\#3873, Cell Signaling).

\section{Statistical analysis}

Each experiment in this study was repeated at least three times. Data are means \pm SD. Statistical analyses were performed with Student's $t$-test for pairwise comparisons using GraphPad Prism 5 (GraphPad Software, La Jolla, CA, USA). $p$ values less than 0.05 were considered significant.

\section{Statement of ethics}

All procedures conducted during this study were approved by the Sookmyung Women's University School Institutional Animal Care and Use Committees.

\section{RESULTS}

\section{Down-regulated $\mathrm{FOXO3}$ promotes ROS accumulation and cyst growth}

Oxidative stress is known to contribute to ADPKD progression (Andries et al., 2019). To confirm that accumulation of ROS progressively occurred in an ADPKD mouse model, we assessed the expression of 8-hydroxy-2-deoxyguanosine (8-OHdG), a marker of oxidative DNA damage, by immunohistochemical staining. In kidney tissues obtained from $P k d 1^{\text {flf: }}$ HoxB7-Cre mice, it was observed that the expression of 8-OHdG gradually increased in the cyst-lining cells as the disease progressed. In contrast, 8-OHdG expression was not observed in kidney tissues of $P k d 1^{1 / f}$ mice on postnatal days 3 , 7, and 13 (Fig. 1A).

The FOXO protein family is known to regulate cellular antioxidant defenses (Klotz et al., 2015), notably FOXO3, which 
A
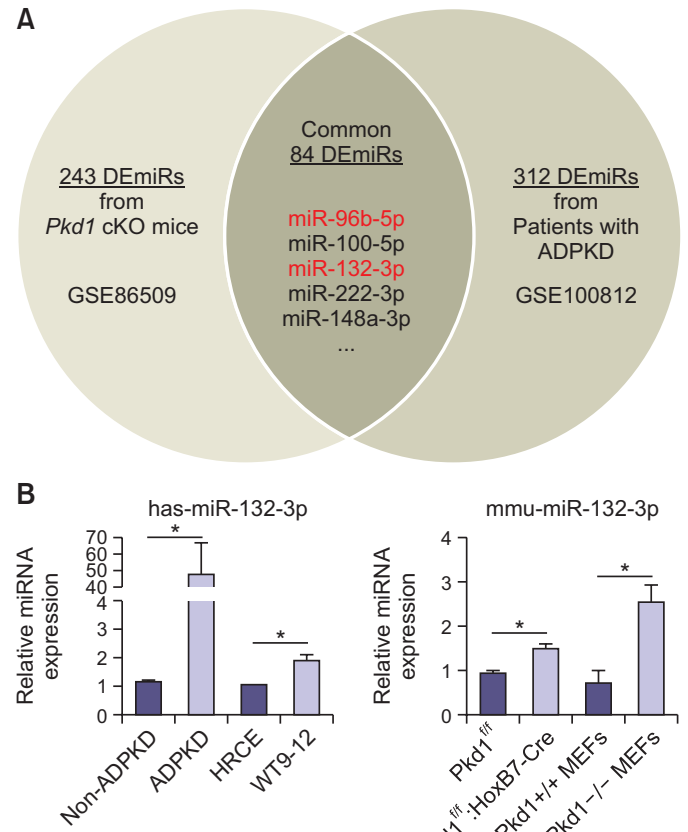

D

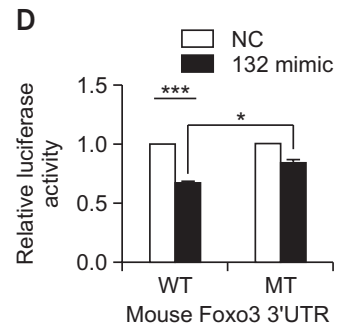

G
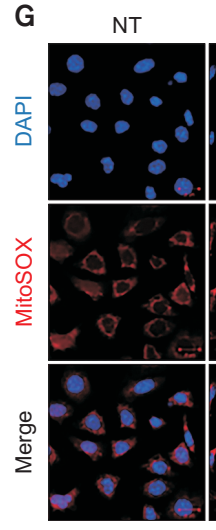

$\mathrm{CoCl}_{2}$

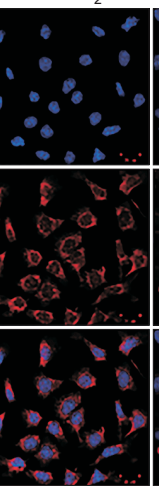

mmu-miR-132-3p
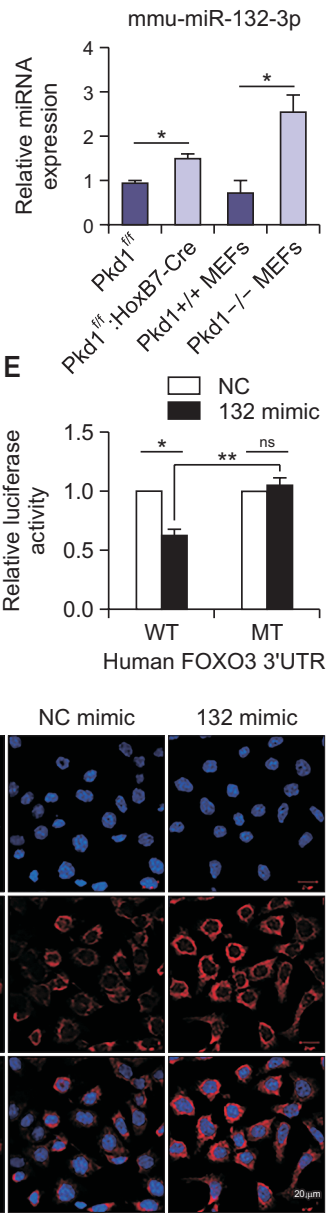

C

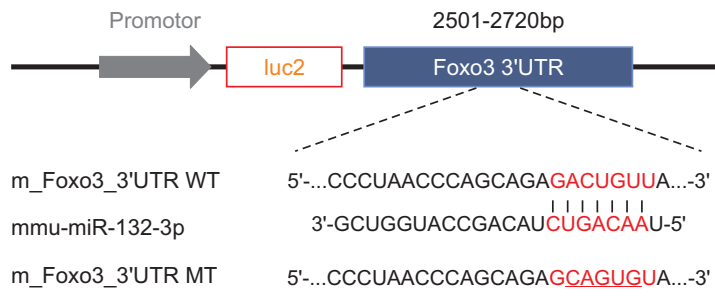

Promotor

2522-2741bp

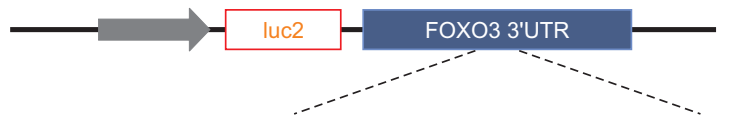

h_FOXO3_3'UTR WT

5'-...UCCUAACCCAGCAGAGACUGUUA....-3'

hsa-miR-132-3p

3'-GCUGGUACCGACAUCU'I'GACÁÁU-5'

h_FOXO3_3'UTR MT

$5^{\prime}-\ldots$ UCCUAACCCAGCAGAGCAGUGUA...-3'
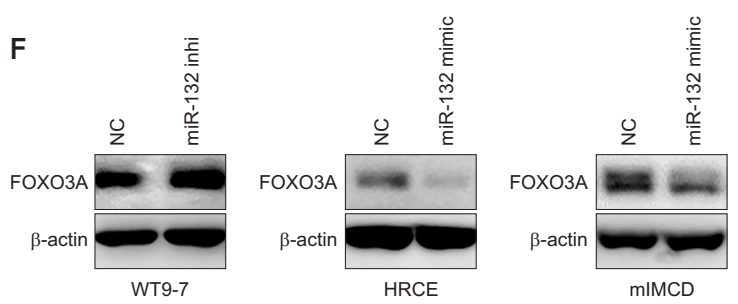

$\mathrm{H}$
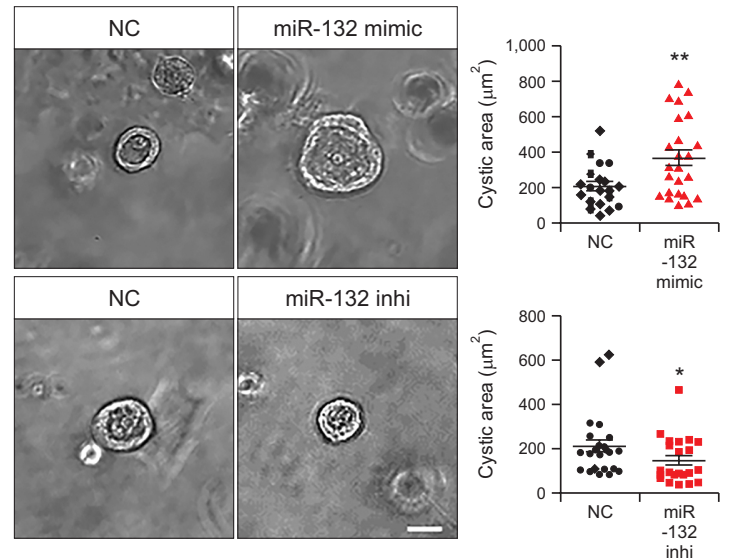

Fig. 2. miR-132-3p is up-regulated and increase mitochondrial superoxide levels by directly targeting FOXO3 in ADPKD. (A) Integrated analysis of miRNA-seq data from $P k d 1^{f f f}:$ HoxB7-Cre mice and microarray data from ADPKD patients. In total, there were 243 differentially expressed miRNAs (DEmiRs) in the miRNA-seq dataset and 312 DEmiRs in the microarray dataset; 84 of these DEmiRs were common to both datasets. (B) Expression validation of candidate miRNAs using qRT-PCR in the kidney tissue of $P k d 1^{f / f}$ and $P k d 1^{\mathrm{fff}}: \mathrm{HoxB7}-\mathrm{Cre}$ mice, $P k d 1+/+$ and Pkd1-/- mouse embryonic fibroblasts (MEFs), kidney tissues from non-ADPKD and ADPKD patients, and HRCE and WT97 cell lines. HRCE is a human renal cortical epithelial cell line isolated from normal human kidney tissue, and WT9-7 is a proximal cortical tubule epithelial cell line isolated from the renal cysts of a patient with ADPKD. (C) Sequence alignment of the wild type (WT) and mutant (MT) FOXO3 3'-UTR in the luciferase constructs with miR-132-3p. The red sequence in the WT construct indicates the predicted target site of miR-132-3p. (D, E) Relative luciferase activity of the 3'-UTR luciferase constructs after co-transfection with either a miR-132-3p mimic (132 mimic) or negative control (NC) in (D) mIMCD cells and (E) HEK293T cells. (F) FOXO3 protein expression in the WT9-7 cell line after transfection with a miR-132-3p inhibitor and in the HRCE and mIMCD cell lines after transfection with miR-132-3p mimics by western blotting. (G) MitoSOX staining to detect mitochondrial superoxide $\left(\mathrm{O}_{2}^{-}\right)$levels by confocal microscopy. IMCD cells were transfected with $30 \mathrm{nM}$ miR-132$3 p$ mimic. $\mathrm{CoCl}_{2}$ was used as a positive control. NT, non-treated. $(\mathrm{H})$ Cyst formation in mIMCD cells transfected with miR-132-3p mimics or inhibitor and NC miR in a Matrigel 3D culture system. Individual cyst sizes were assessed after 6 days of 3D culture. Scale bar, $50 \mu \mathrm{m}$. Data are the mean \pm SD of triplicate samples from three independent experiments. ${ }^{*} p<0.05,{ }^{* *} p<0.01,{ }^{* * *} p<0.001$. 
A

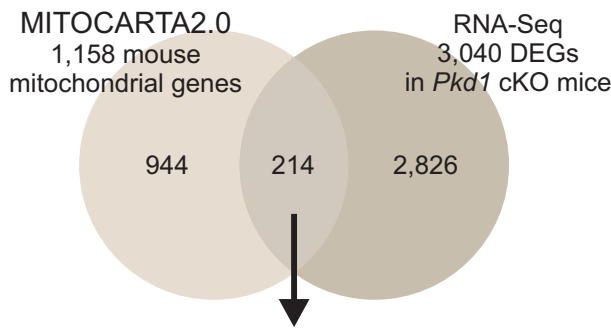

Downregulation $P k d 1$ cKO model

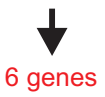

B

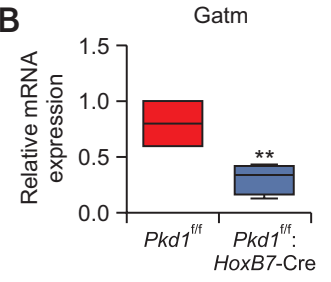

D

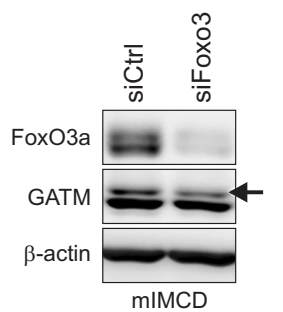

C
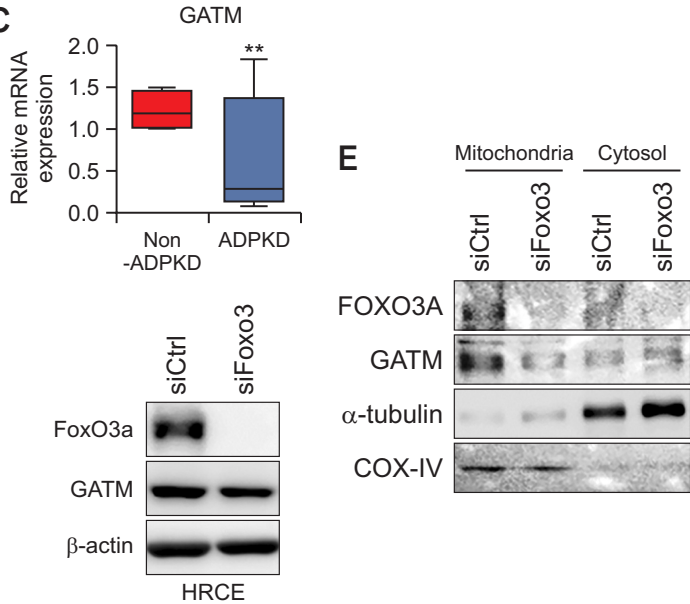

FOXO3A

GATM

$\alpha$-tubulin

COX-IV

G
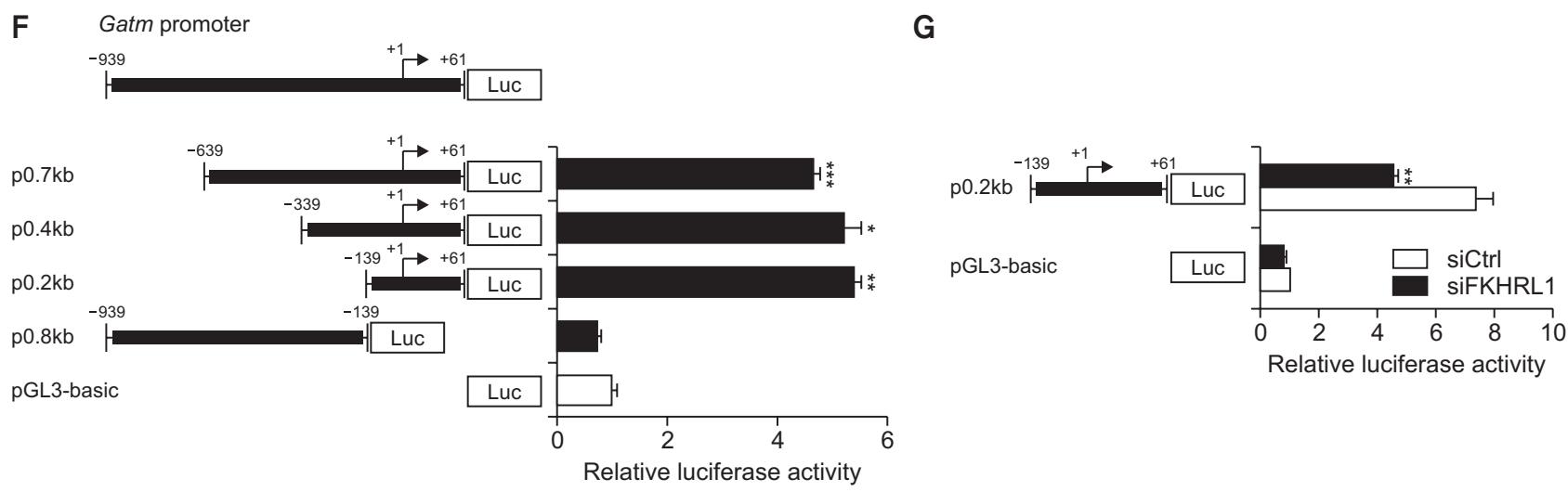

Fig. 3. Gatm is down-regulated in ADPKD and regulated by FOXO3. (A) Schematic of the comparative analysis of mitochondrial genes and differentially expressed genes (DEGs) in Pkd1 cKO mice for the identification of differentially expressed mitochondrial genes. (B, C) Validation of the mRNA expression levels of Gatm using qRT-PCR in the (B) kidney tissues of $P k d 1^{\text {t/f }}$ and $P k d 1^{\text {t/f }}:$ HoxB7-Cre mice and (C) non-ADPKD and ADPKD patients. (D) The protein expression levels of GATM in mIMCD and HRCE cells transfected with Foxo3 or control siRNA $(20 \mathrm{nM})$ by western blotting. (E) GATM protein expression in the mitochondrial and the cytosolic fractions of mIMCD cells transfected with Foxo3 or control siRNA ( $20 \mathrm{nM}$ ) by western blotting. (F) Relative luciferase activity of four Gatm promoter region constructs in mIMCD cells. Several deletion constructs of the Gatm promoter region inserted into the pGL3-base luciferase reporter vector are shown on the right. (G) Relative luciferase activity of the p0.2 kb construct in mIMCD cells transfected with Foxo3 or control siRNA. Data are the mean \pm SD of triplicate samples from three independent experiments. ${ }^{*} p<0.05,{ }^{* *} p<0.01,{ }^{* * *} p<0.001$.

is required for the regulation of oxidative stress (Marinkovic et al., 2007). To determine its association with oxidative stress in ADPKD models, we analyzed the transcript and protein levels of Foxo3 in the kidneys of PKD mice. The expression levels of Foxo3 were revealed to be reduced in Pkd1 cKO mouse tissues (Fig. 1B-1D). Since mitochondria are major intracellular sources of ROS with superoxide as the predominant $\mathrm{ROS}$, mitochondrial $\mathrm{O}_{2}{ }^{-}$generation was measured using the mitochondria-targeting probe MitoSOX Red. Mitochondrial superoxide levels markedly increased in downregulated Foxo3 in mouse inner medullary collecting duct (IMCD) cells compared to the negative controls (Fig. 1E). We further investigated whether down or up regulation of Foxo3 and consequent oxidative damage might affect ADPKD progression. The evident pathology of ADPKD is that numerous fluid-filled cysts are developed from renal tubular cells. Therefore, cyst growth in IMCD cells treated with siFoxo3, Foxo3 construct or $\mathrm{H}_{2} \mathrm{O}_{2}$ was measured in a three-dimensional (3D) cell culture system (Fig. 1F). Inducing oxidative damage using $\mathrm{H}_{2} \mathrm{O}_{2}$ and repression of Foxo3 significantly accelerated cyst progression. In contrast, overexpression of Foxo3 alleviated cyst formation. These results suggest that reduced Foxo3 affects accumulation of ROS, and finally accelerates cyst expansion in ADPKD.

\section{miR-132-3p is upregulated in mice and humans with ADPKD}

To identify the regulator of FOXO3a in ADPKD models, we performed an integrated analysis of miRNA sequencing data from $P k d 1^{\mathrm{fff}}$ :HoxB7-Cre mice and microarray data from patients with ADPKD. Previous studies performed miRNAs in kidney tissues from $P k d 1^{\text {fff }}$ :HoxB7-Cre mice with those from $P k d 1^{\text {fff }}$ controls by miRNA sequencing (GSE86509) and miRNAs in tissues from patients with ADPKD with non-ADPKD patients by microarray (GSE100812) (Woo et al., 2017; Kim et al., 2019). Differentially expressed miRNAs (DEmiR) had values of $p<0.05$ and the gene expression for such miRNAs was found to exhibit a 2-fold change in mice models or a 1.5fold change in patients with ADPKD. We identified 84 miRNAs common to both Pkd1 cKO mice and patients with ADPKD, from a total of 243 DEmiRs in Pkd1 Pkd1 cKO mice and 312 

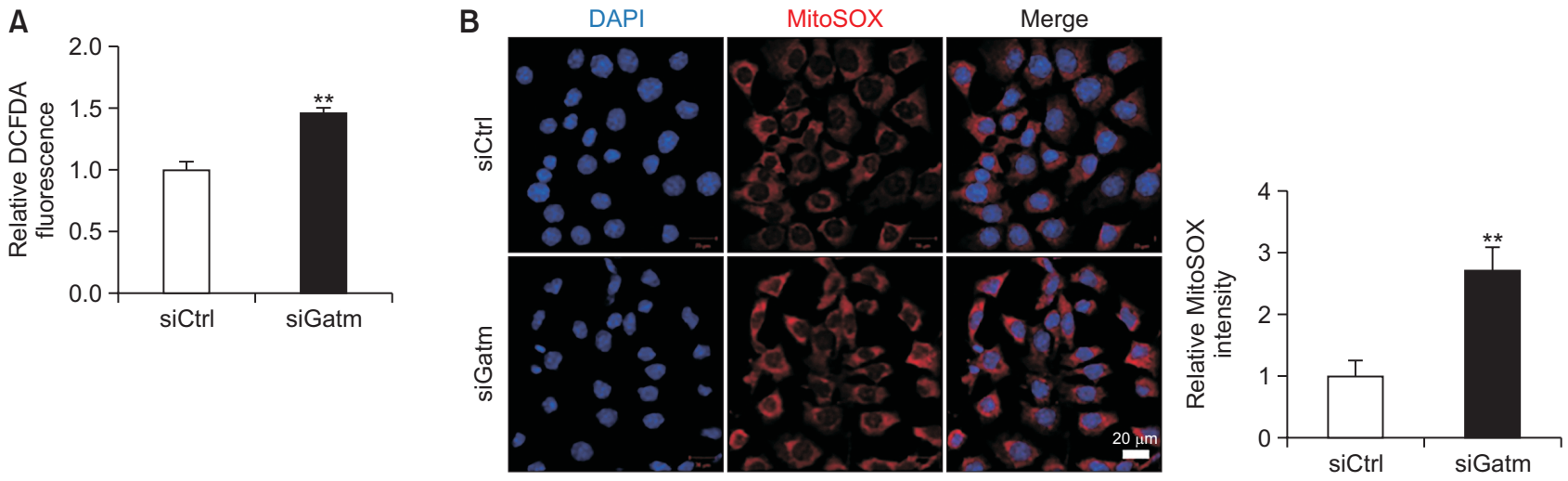

C

Mito tracker green

MitosoX red

Merge
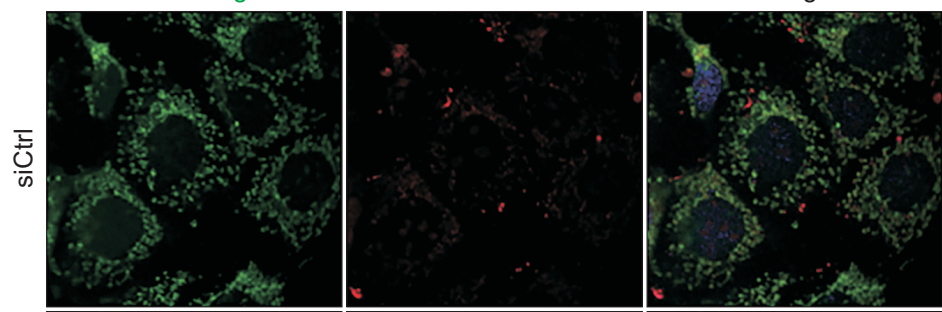

D
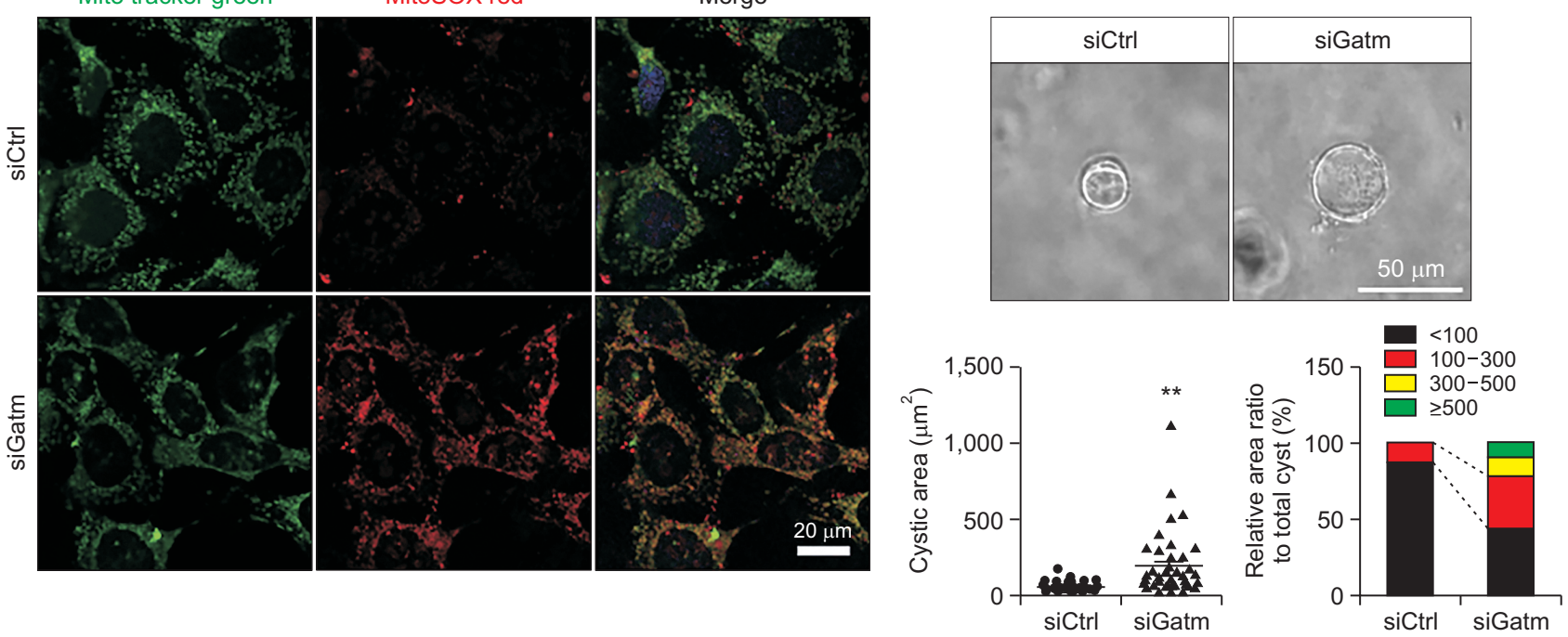

Fig. 4. Suppression of Gatm promotes cyst expansion by increasing ROS. (A) 2',7'-dichlorofluorescein diacetate (DCFDA) was used to measure cellular ROS activity, including hydroxyl, peroxyl and other ROS. The intensity of DCFDA was measured using a microplate reader. (B) MitoSOX staining to detect mitochondrial superoxide $\left(\mathrm{O}_{2}^{-}\right)$levels by confocal microscopy. IMCD cells were transfected with Gatm siRNA $(20 \mathrm{nM})$. $\mathrm{CoCl}_{2}$ was used as a positive control. NT, non-treated. (C) Co-staining with MitoSOX Red and MitoTracker Green, which accumulates in active mitochondria. Images were obtained by confocal microscopy at $400 \times$ magnification and $1.5 \times$ zoom. (D) Cyst formation in control or Gatm siRNA treated IMCD cells in a Matrigel 3D culture system. Cyst area was measured after 4 days in 3D culture. Data are presented as the mean \pm SD of triplicate samples from three independent experiments. ${ }^{* *} p<0.01$.

DEmiRs in patients with ADPKD (Fig. 2A). Among the common miRNAs, only two were capable of binding to the Foxo3 3'-UTR, namely miR-96-5p and miR-132-3p. Next, to validate the expression of the two candidate miRNAs, we assessed their expression levels in kidney tissues and cells from mouse models and in patients with ADPKD by qRT-PCR. Interestingly, only miR-132-3p was observed to be significantly expressed in both mice and humans with ADPKD (Fig. 2B, Supplementary Fig. 1). To determine whether miR-132-3p directly inhibited FOXO3 expression in both human and mouse renal cell lines, we performed a luciferase reporter assay using a reporter construct with either the mouse Foxo3 3'-UTR or human FOXO3 3'-UTR, where both contain a miR-132-3p-binding site (Fig. $2 \mathrm{C})$. Each construct was co-transfected with either a miR-132$3 p$ mimic or negative control miRNA (NC) into IMCD cells or human embryonic kidney 293T (HEK293T) cells. As expected, we found that miR-132-3p directly bound to the 3'-UTR of both FOXO3 and FoxO3, thereby negatively regulating translation
(Fig. 2D-2F). Next, we examined whether increased miR-132$3 p$ expression and the consequent reduction of Foxo3 expression were responsible for oxidative stress in ADPKD. It was found that mitochondrial superoxide increased during ectopic expression of miR-132 in IMCD cells compared to that in the negative control (Fig. 2G). Representative photographs show that overexpression of miR-132-3p significantly promoted cyst growth compared to cells transfected with NC in the 3D culture; in contrast, inhibition of miR-132 alleviated cyst formation (Fig. $2 \mathrm{H}$ ). These observations suggest that miR-132-3p causes oxidative stress and accelerates cyst growth by inhibiting Foxo3 in ADPKD.

\section{Gatm is the mediator of Foxo3 in ADPKD}

FOXO3 is involved in reactive oxygen metabolism by regulating mitochondrial gene expression (Ferber et al., 2012). To explore the mechanisms associated with FOXO3 in ADPKD, we screened mitochondrial genes regulated by $\mathrm{FOXO} 3 \mathrm{ac}-$ 
cordingly. We performed integrative analysis between 1158 mouse mitochondrial genes from the Broad Institute (Calvo et al., 2016) and the 3040 differentially expressed genes (DEGs) in $P k d 1^{f f f}:$ HoxB7-Cre mice obtained by RNA-seq data from GSE86509, which had been done in our previous study (Woo et al., 2017). It finally identified 214 common genes between them (Fig. 3A). Mitochondrial genes downregulated with $\log F C$ values less than -1.3 were selected, thereby revealing 6 mitochondrial genes (Aadat, Lars2, Gatm, Hmgcs2, Acss1, and Aldh1/1) with reduced expression in Pkd1 cKO mice (Supplementary Table 1). To validate the expression of these genes in ADPKD, the mRNA expression levels of the 6 selected genes were confirmed in kidney tissues of PKD mice and patients with ADPKD using qRT-PCR. All 6 genes were significantly reduced in $P k d 1^{\mathrm{fff}}: \mathrm{HoxB} 7-\mathrm{Cre}$ mice when compared to the levels in $P k d 1^{\mathrm{flf}}$ mice (Fig. 3B, Supplementary Fig. 2A). Notably, only GATM were significantly reduced in ADPKD patients compared to the levels in non-ADPKD patients (Fig. 3C, Supplementary Fig. 2B). Furthermore, we used western blotting to assess the expressed protein levels of GATM in cells transfected with FOXO3 siRNA. GATM was decreased by FOXO3 knockdown in both mIMCD and HRCE cells (Fig. 3D). Additionally, to confirm that the inhibition of FOXO3 reduced GATM expression in mitochondria, the mitochondrial and cytoplasmic fractions of mIMCD cells transfected with FKHRL1 siRNA were isolated and analyzed by western blotting, which revealed that inhibition of FOXO3 decreased GATM expression in the mitochondria of mIMCD cells (Fig. 3E). These results suggest that GATM is regulated by $\mathrm{FOXO} 3$ in the mitochondria.

We then investigated whether FOXO3 bound to specific DNA sequences of the Gatm promoter to regulate transcription. Several deletion constructs of the Gatm promoter region were cloned into the pGL3-basic luciferase reporter vector and transfected into mIMCD cells. Interestingly, luciferase activity was significantly higher in cells transfected with vectors containing constructs of the $0.2-\mathrm{kb}$ region of the Gatm promoter compared to the levels in the control, but not in cells transfected with $\mathrm{p} 0.8 \mathrm{~kb}$ which lacked the $0.2-\mathrm{kb}$ region (Fig. $3 F$ ). To confirm whether the $0.2-k b$ region was crucial for promoter activity, we performed a luciferase activity assay under downregulated Foxo3 expression conditions. Knockdown of Foxo3 significantly reduced the promoter activity of the $0.2-\mathrm{kb}$ construct-containing vector compared to the control (Fig. 3G). These data indicate that GATM is regulated by the transcription factor FOXO3 in ADPKD.

\section{Inhibition of Gatm exacerbates cyst enlargement and oxidative stress in ADPKD}

The effect of Gatm on oxidative stress in ADPKD was verified using 2,7-dichlorofluorescein diacetate (DCFDA), which is used to measure cellular ROS, including hydroxyl, peroxyl, and other ROS. Results of the experiment showed that knockdown of Gatm significantly elevated DCFDA fluorescence intensity (Fig. 4A). More specifically, Gatm knockdown significantly increased mitochondrial superoxide levels (Fig. 4B). Additionally, this Gatm-mediated increase in superoxide levels was confirmed by co-staining with MitoSOX Red and MitoTracker Green, both of which accumulate in active mitochondria (Fig. 4C). To investigate the effect of Gatm on cyst formation in ADPKD, Gatm-knocked down IMCD cells were analyzed in 3D cell culture, where inhibition of Gatm in IMCD

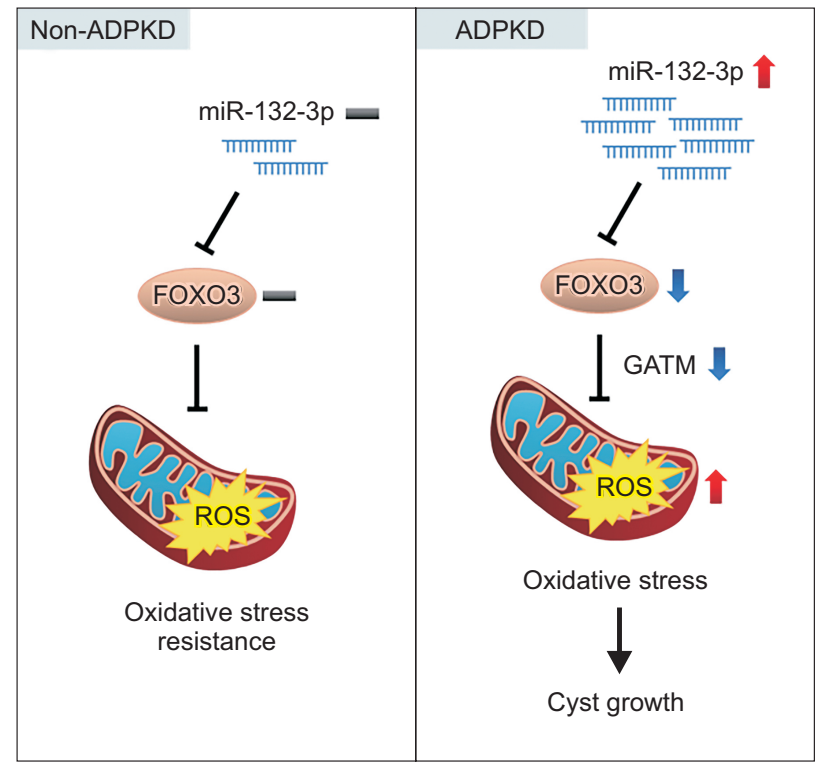

Fig. 5. Schematic of the proposed novel mechanism of ADPKD progression. miR-132-3p is up-regulated in ADPKD. Increased miR-132-3p levels inhibit Foxo3 and subsequently down-regulate Gatm, leading to elevated ROS levels and oxidative stress, which promotes cyst growth in ADPKD.

cells significantly promoted cyst progression (Fig. 4D). Specifically, the proportion of cysts that grew from $100 \mu \mathrm{m}^{2}$ to 300 $\mu \mathrm{m}^{2}$ was $12.5 \%$ in the control group, while it was at $34.9 \%$ in Gatm-knocked down IMCD cells. Cysts greater than $300 \mu \mathrm{m}^{2}$ were not observed in the control; however, it should be noted that $20.9 \%$ of the total cysts in IMCD cells transfected with siGatm were greater than $300 \mu \mathrm{m}^{2}$. Taken together, these data suggest that miR-132-3p promotes oxidative stress and cyst expansion in ADPKD by inhibition of Foxo3 and Gatm.

\section{DISCUSSION}

It has been reported that oxidative stress appears in the early stages of ADPKD and worsens with disease progression (Andries et al., 2019). Recent studies have suggested that oxidative DNA damage caused by mitochondrial superoxide may cause mutations in PKD-related genes (Ishimoto et al., 2017). Additionally, it has also been reported that tolvaptan, the sole treatment for ADPKD, activates the antioxidant pathway (Fujiki et al., 2019). Although the importance of oxidative stress in ADPKD has been highlighted, its molecular mechanism has not been clarified. To discover an effective treatment for ADPKD, it is necessary to understand the mechanism of oxidative stress in ADPKD. In this study, we identified a novel mechanism, miR-132-3p-Foxo3a-Gatm, associated with oxidative stress in ADPKD.

The respective roles of miR132 and Foxo3 in chronic kidney disease (CKD) following renal injury have been recently reported. In kidneys with ischemic injury, chronic hypoxia developed and it accumulated $\mathrm{FOXO} 3$ by inhibiting its degradation. In addition, hypoxia-activated HIF1 $\alpha$ controlled the late phase of renal tubular repair by FOXO3 activation, which finally contributed to functional recovery (Li et al., 2019; Lin, 2020). 
Severe renal injury leads to CKD when the repair processes fail to completely recover, and progressive interstitial fibrosis is involved during its transition. MiRNAs profiling in fibrotic kidneys revealed a specific increase of miR132 level in response to ischemia-reperfusion injury in mice. MiR132 affected proliferative capacity of pericyte-derived myofibroblasts by coordinately regulating TGF- $\beta$ and STAT3/ERK pathways (Bijkerk et al., 2016). In these regards, targeting either Foxo3 or miR132 has been potentially considered as potential CKD therapies. However, the direct correlations of miR132 with Foxo3 and Gatm following oxidative stress have not been identified until now. Herein, we first verify the functional mechanism of miR132/Foxo3/Gatm axis in renal cystic model.

FOXO3 is a negative regulator of mitochondrial ROS which mediates the defense against oxidative stress via regulation of ROS-scavenging enzymes (Liu et al., 2005). However the function of Foxo3 has not been elucidated in ADPKD. Based on our results, oxidative stress is increased while Foxo3 is reduced over time in ADPKD models. Furthermore, several studies have reported that $\mathrm{FOXO} 3$ is involved in reactive oxygen metabolism via regulation of mitochondrial gene expression, and that regulation of the mitochondrial structure and function may be important factors which regulate ROS production (Ferber et al., 2012). We observed that Foxo3 transcriptionally regulated mitochondrial gene Gatm. It encodes mitochondrial enzyme which is involved in creatine biosynthesis via catalyzing the immediate precursor of creatine, guanidinoacetic acid (Humm et al., 1997). In the field of kidney disease research, clinical case studies have revealed monoalleic mutations observed in autosomal dominant renal Fanconi syndrome and progressive kidney failure (Reichold et al., 2018). In our observations, the expression of Gatm was significantly downregulated in both kidney tissues from $P k d 1^{\text {ftf }}$ :HoxB7-Cre mice and ADPKD patients. Based on these findings, we suggest that Gatm is involved in a mechanism where Foxo3 influences oxidative stress.

In our previous study, we reported the miRNAs that were differentially expressed in PKD model mice and human ADPKD patients by miRNA sequencing and microarray analysis, respectively, and reported that abnormal miRNA expression was closely associated with ADPKD (Li et al., 2017; Kim et al., 2019). In the present study, we aimed to identify the miRNAs associated with ADPKD and elucidate the mechanisms responsible for cyst formation, the major phenotype of the disease, in order to identify novel therapeutic targets. We performed an integrated analysis of the differentially expressed miRNAs obtained by miRNA sequencing analysis of PKD mouse models and by microarray analysis of ADPKD patients and validated the observed expression patterns. Our data showed that miR-132-3p was significantly upregulated and consequently suppressed Foxo3 transcription in kidney tissues and cell lines from mice and humans with ADPKD, suggesting that miR-132-3p was strongly associated with ADPKD.

To verify that miR-132-3p affected ROS accumulation, we assessed the expression of miR-132-3p in the ubiquinol-cytochrome $c$ reductase binding protein (UQCRB) mutant cell line, which showed increased mitochondrial ROS (Kim et al., 2018). As expected, the expression of miR-132-3p in the UQCRB mutant cell line was not significantly different from that in the HEK293 cell line (Supplementary Fig. 3). These results suggest that upregulation of miR-132-3p precedes ROS production.
Based on the observed results of this study, we present a new mechanism of cystogenesis, in which increased miR-132$3 p$ expression inhibits Foxo3 and reduces Gatm expression, thereby leading to elevated ROS levels and oxidative stress, subsequently promoting cyst growth in ADPKD (Fig. 5). Furthermore, we suggest that the interaction between miR-132$3 p$ and its target play a crucial role in cystogenesis in ADPKD by mediating oxidative stress. The miR-132-3p-Foxo3-Gatm axis may be a novel therapeutic target and prognostic marker for ADPKD.

\section{CONFLICT OF INTEREST}

The authors declare that they have no conflicts of interest.

\section{ACKNOWLEDGMENTS}

This work was supported by grants from the Collaborative Genome Program for Fostering New Post-Genome Industry of the NRF [2014M3C9A2067613] and the Basic Science Program [2016R1A5A1011974].

\section{REFERENCES}

Andries, A., Daenen, K., Jouret, F., Bammens, B., Mekahli, D. and Van Schepdael, A. (2019) Oxidative stress in autosomal dominant polycystic kidney disease: player and/or early predictor for disease progression? Pediatr. Nephrol. 34, 993-1008.

Bijkerk, R., de Bruin, R. G., van Solingen, C., van Gils, J. M., Duijs, J. M., van der Veer, E. P., Rabelink, T. J., Humphreys, B. D. and van Zonneveld, A. J. (2016) Silencing of microRNA-132 reduces renal fibrosis by selectively inhibiting myofibroblast proliferation. Kidney Int. 89, 1268-1280.

Calnan, D. R. and Brunet, A. (2008) The FoxO code. Oncogene 27, 2276-2288.

Calvo, S. E., Clauser, K. R. and Mootha, V. K. (2016) MitoCarta2.0: an updated inventory of mammalian mitochondrial proteins. Nucleic Acids Res. 44, D1251-D1257.

Ferber, E. C., Peck, B., Delpuech, O., Bell, G. P., East, P. and Schulze, A. (2012) FOXO3a regulates reactive oxygen metabolism by inhibiting mitochondrial gene expression. Cell Death Differ. 19, 968979.

Fujiki, T., Ando, F., Murakami, K., Isobe, K., Mori, T., Susa, K., Nomura, N., Sohara, E., Rai, T. and Uchida, S. (2019) Tolvaptan activates the Nrf2/HO-1 antioxidant pathway through PERK phosphorylation. Sci. Rep. 9, 9245.

Gansevoort, R. T., Arici, M., Benzing, T., Birn, H., Capasso, G., Covic, A., Devuyst, O., Drechsler, C., Eckardt, K. U., Emma, F., Knebelmann, B., Le Meur, Y., Massy, Z. A., Ong, A. C., Ortiz, A., Schaefer, F., Torra, R., Vanholder, R., Więcek, A., Zoccali, C. and Van Biesen, W. (2016) Recommendations for the use of tolvaptan in autosomal dominant polycystic kidney disease: a position statement on behalf of the ERA-EDTA Working Groups on Inherited Kidney Disorders and European Renal Best Practice. Nephrol. Dial. Transplant. 31, 337-348.

Hagenbuchner, J., Kuznetsov, A., Hermann, M., Hausott, B., Obexer, P. and Ausserlechner, M. J. (2012) FOXO3-induced reactive oxygen species are regulated by BCL2L11 (Bim) and SESN3. J. Cell Sci. 125, 1191-1203.

Ham, H. J., Park, J. W. and Bae, Y. S. (2019) Defect of SIRT1-FoxO3a axis is associated with the production of reactive oxygen species during protein kinase CK2 downregulation-mediated cellular senescence and nematode aging. BMB Rep. 52, 265-270.

Humm, A., Fritsche, E., Steinbacher, S. and Huber, R. (1997) Crystal 
structure and mechanism of human L-arginine:glycine amidinotransferase: a mitochondrial enzyme involved in creatine biosynthesis. EMBO J. 16, 3373-3385.

Igarashi, P. and Somlo, S. (2007) Polycystic kidney disease. J. Am. Soc. Nephrol. 18, 1371-1373.

Ishimoto, Y., Inagi, R., Yoshihara, D., Kugita, M., Nagao, S., Shimizu, A., Takeda, N., Wake, M., Honda, K., Zhou, J. and Nangaku, M. (2017) Mitochondrial abnormality facilitates cyst formation in autosomal dominant polycystic kidney disease. Mol. Cell. Biol. 37 , e00337-17.

Kim, D. Y., Woo, Y. M., Lee, S., Oh, S., Shin, Y., Shin, J. O., Park, E. Y., Ko, J. Y., Lee, E. J., Bok, J., Yoo, K. H. and Park, J. H. (2019) Impact of miR-192 and miR-194 on cyst enlargement through EMT in autosomal dominant polycystic kidney disease. FASEB J. 33, 2870-2884..

Kim, J. E., Hong, J. W., Lee, H. S., Kim, W., Lim, J., Cho, Y. S. and Kwon, H. J. (2018) Hsa-miR-10a-5p downregulation in mutant UQCRB-expressing cells promotes the cholesterol biosynthesis pathway. Sci. Rep. 8, 12407

Klotz, L. O., Sanchez-Ramos, C., Prieto-Arroyo, I., Urbanek, P., Steinbrenner, H. and Monsalve, M. (2015) Redox regulation of FoxO transcription factors. Redox Biol. 6, 51-72.

Kops, G. J., Dansen, T. B., Polderman, P. E., Saarloos, I., Wirtz, K. W., Coffer, P. J., Huang, T. T., Bos, J. L., Medema, R. H. and Burgering, B. M. (2002) Forkhead transcription factor FOXO3a protects quiescent cells from oxidative stress. Nature 419, 316-321.

Li, L., Kang, H., Zhang, Q., D'Agati, V. D., Al-Awqati, Q. and Lin, F. (2019) FoxO3 activation in hypoxic tubules prevents chronic kidney disease. J. Clin. Invest. 129, 2374-2389.

Li, Y., Li, J., Woo, Y. M., Shen, Z., Yao, H., Cai, Y., Lin, M. C. and Poon, W. S. (2017) Enhanced expression of Vastatin inhibits angiogenesis and prolongs survival in murine orthotopic glioblastoma model. BMC Cancer 17, 126.

Lin, F. (2020) Molecular regulation and function of FoxO3 in chronic kidney disease. Curr. Opin. Nephrol. Hypertens 29, 439-445.

Liu, J. W., Chandra, D., Rudd, M. D., Butler, A. P., Pallotta, V., Brown, D., Coffer, P. J. and Tang, D. G. (2005) Induction of prosurvival molecules by apoptotic stimuli: involvement of FOXO3a and ROS. Oncogene 24, 2020-2031.

Marinkovic, D., Zhang, X., Yalcin, S., Luciano, J. P., Brugnara, C., Huber, T. and Ghaffari, S. (2007) Foxo3 is required for the regulation of oxidative stress in erythropoiesis. J. Clin. Invest. 117, 2133-2144.

Mochizuki, T., Wu, G., Hayashi, T., Xenophontos, S.L., Veldhuisen, B.,
Saris, J. J., Reynolds, D. M., Cai, Y., Gabow, P. A., Pierides, A., Kimberling, W. J., Breuning, M. H., Deltas, C. C., Peters, D. J. and Somlo, S. (1996) PKD2, a gene for polycystic kidney disease that encodes an integral membrane protein. Science 272, 1339-1342.

Peters, D. J. and Sandkuijl, L. A. (1992) Genetic heterogeneity of polycystic kidney disease in Europe. Contrib. Nephrol. 97, 128-139.

Reichold, M., Klootwijk, E. D., Reinders, J., Otto, E. A., Milani, M., Broeker, C., Laing, C., Wiesner, J., Devi, S., Zhou, W., Schmitt, R., Tegtmeier, I., Sterner, C., Doellerer, H., Renner, K., Oefner, P. J., Dettmer, K., Simbuerger, J. M., Witzgall, R., Stanescu, H. C., Dumitriu, S., lancu, D., Patel, V., Mozere, M., Tekman, M., Jaureguiberry, G., Issler, N., Kesselheim, A., Walsh, S. B., Gale, D. P., Howie, A. J., Martins, J. R., Hall, A. M., Kasgharian, M., O’Brien, K., Ferreira, C. R., Atwal, P. S., Jain, M., Hammers, A., Charles-Edwards, G., Choe, C. U., Isbrandt, D., Cebrian-Serrano, A., Davies, B., Sandford, R. N., Pugh, C., Konecki, D. S., Povey, S., Bockenhauer, D., Lichter-Konecki, U., Gahl, W. A., Unwin, R. J., Warth, R. and Kleta, R. (2018) Glycine amidinotransferase (GATM), renal fanconi syndrome, and kidney failure. J. Am. Soc. Nephrol. 29, 1849-1858.

Torres, V. E., Chapman, A. B., Devuyst, O., Gansevoort, R. T., Grantham, J. J., Higashihara, E., Perrone, R. D., Krasa, H. B., Ouyang, J. and Czerwiec, F. S.; TEMPO 3:4 Trial Investigators (2012) Tolvaptan in patients with autosomal dominant polycystic kidney disease. N. Engl. J. Med. 367, 2407-2418.

Tzivion, G., Dobson, M. and Ramakrishnan, G. (2011) FoxO transcription factors; regulation by AKT and 14-3-3 proteins. Biochim. Biophys. Acta 1813, 1938-1945.

van der Horst, A. and Burgering, B. M. (2007) Stressing the role of FoxO proteins in lifespan and disease. Nat. Rev. Mol. Cell Biol. 8, 440-450.

Woo, Y. M., Bae, J. B., Oh, Y. H., Lee, Y. G., Lee, M. J., Park, E. Y., Choi, J. K., Lee, S., Shin, Y., Lyu, J., Jung, H. Y., Lee, Y. S., Hwang, Y. H., Kim, Y. J. and Park, J. H. (2014) Genome-wide methylation profiling of ADPKD identified epigenetically regulated genes associated with renal cyst development. Hum. Genet. 133, 281-297.

Woo, Y. M., Kim, D. Y., Koo, N. J., Kim, Y. M., Lee, S., Ko, J. Y., Shin, Y., Kim, B. H., Mun, H., Choi, S., Lee, E. J., Shin, J. O., Park, E. Y., Bok, J. and Park, J. H. (2017) Profiling of miRNAs and target genes related to cystogenesis in ADPKD mouse models. Sci. Rep. 7, 14151.

Zhang, J., Wang, X., Vikash, V., Ye, Q., Wu, D., Liu, Y. and Dong, W. (2016) ROS and ROS-mediated cellular signaling. Oxid. Med. Cell. Longev. 2016, 4350965. 